\title{
Anestetizzare il conflitto? Slittamenti di significato di due casi brasiliani di Bilancio Partecipativo
}

\author{
di Giovanni Allegretti, Geraldo Campos e Fabio Siqueira ${ }^{*}$
}

\begin{abstract}
Il conflitto genera interesse attorno al trattamento dei problemi collettivi [...] spinge gli attori a definire meglio le loro posizioni, mobilitando [...] risorse, anche di tipo conoscitivo, articolando e arricchendo la definizione del problema (P. Fareri 2009, Rallentare. Il disegno delle politiche urbane, Franco Angeli, Milano p. 217).

La democrazia è il governo per mezzo della discussione, ma funziona solo se si riesce a far smettere la gente di discutere (C. Attlee 1957, in A. Sen 2010, L'idea di giustizia, Mondadori, Milano, p. 330).
\end{abstract}

\section{Introduzione}

Nell'ultimo ventennio, molte esperienze di gestione locale partecipativa in Brasile hanno rappresentato un punto di riferimento per la letteratura e la militanza politica di altri continenti (Douglas e Friedmann 1997; Abers, 2000; Bobbio 2002; Fung e Wright 2003; Santos 2004; Avritzer 2006; Hwang 2008). La loro complessa articolazione si è imposta come elemento epistemologico centrale per comprendere la radicalità dei loro orizzonti e l'elevata capacità di trasmettere con chiarezza su quali cardini imperniare il

* Giovanni Allegretti è ricercatore presso il Centro di Studi Sociali dell'Università di Coimbra, dove coordina il dottorato "Democrazia nel secolo XXI"; Geraldo Campos è professore alla Escola Superior de Propaganda e Marketing di São Paolo, è stato coordinatore delle Relazioni Internazionali al Comune di São Paolo e co-autore del libro "Democracia Participativa e Redistribuição" (São Paulo, Ed. Xamã, 2008); Fabio Rejaili Siqueira è avvocato, sociologo e ricercatore presso la Universidad de São Paolo. 
rinnovamento delle politiche pubbliche per contribuire al rafforzamento delle istituzioni locali e (attraverso di esse) del senso dello Stato nel suo complesso.

Se la letteratura converge nell'indicare gli anni Ottanta come il "decennio perduto" per l'America Latina, soprattutto in termini di sviluppo economico (Hettne 1996), vi è anche consenso sul fatto che, politicamente, proprio quegli anni hanno prodotto elaborazioni oggi divenute patrimonio comune di tutto il pianeta. Nel caso del Brasile, non va dimenticato che soprattutto dalla promulgazione della Legge sullo "Statuto della Città"1 (che deve struttura e approvazione alla lunga battaglia condotta da migliaia di attori sociali organizzati nel Movimento di Riforma Urbana) - molte sperimentazioni partecipative condotte autonomamente in varie città brasiliane dalla fine degli anni Ottanta hanno trovato il forte appoggio giuridicoistituzionale di livelli superiori dello Stato $^{2}$. Per tale via, si sono imposte come routine di un numero crescente di amministrazioni locali, rafforzando queste ultime davanti alle istituzioni centrali al punto che oggi i comuni brasiliani risultano tra i pochi enti locali al mondo a non aver subito i contraccolpi della crisi finanziaria internazionale. Anzi, hanno spesso visto crescere la centralità del proprio ruolo e sperimentato in minor grado l'asimmetria tra l'attribuzione di competenze, risorse e capacità decisionali con cui altrove prende forma la traduzione dei principi di sussidiarietà verticale nell' articolazione del rapporto tra i diversi livelli istituzionali della democrazia rappresentativa ${ }^{3}$.

Il principale fattore di attrazione dei tentativi di emulazione delle sperimentazioni partecipative compiuti in parti diverse del pianeta è la multidimensionalità dei loro impatti su cultura civica, giustizia distributiva, dinamizzazione dei sistemi istituzionali ed efficacia delle politiche pubbliche, specie se legate all'inclusione sociale o al reinserimento degli insediamenti spontanei nella città di diritto. Un fenomeno di "marginalizzazione cogniti-

Legge n. 10257/2001.

2 Anche Perù (2003) e Rep. Dominicana (2007) hanno optato per la "statuizione normativa" nazionale, a partire da varie sperimentazioni sviluppate in autonomia a livello locale (Jorge 2007).

3 Il "Rapporto sulla finanza locale" della CNM brasiliana del 2009 e il "Rapporto Economia e Finanza Locale 2009" di IFEL/ANCI evidenziano lo scarto tra municipi brasiliani e italiani. Dal 2002 (con il Governo Lula), la "Marcha dos Municipios" organizzata dalla Confederazione dei Comuni brasiliani contro i tagli ai trasferimenti verso i municipi e la mancata cessione di maggiore autonomia ha cambiato ruolo, divenendo spazio rituale in cui ogni anno Governo e Parlamento ascoltano le rivendicazioni dei Comuni, firmando accordi di concertazione costruttiva. Ciò ha garantito al Presidente Paulo Zilkosky (di opposizione al governo) la doppia rielezione all'unanimità. 
va" riguarda, invece, lo scarso impegno tributato dalla letteratura all'analisi delle trasformazioni subite negli ultimi anni da alcuni percorsi partecipativi oggetto in passato di studi approfonditi. Ciò lascia in ombra il mutare delle condizioni di contesto dentro cui tali esperimenti erano maturati.

Se è comprensibile che molti attori politico/istituzionali - o i movimenti legati alla militanza sociale di base - tendano a guardare al "firmamento" delle pratiche partecipative come a uno spazio in cui «le stelle brillano an-cora a lungo dopo la loro morte» ${ }^{4}$ in virtù dei principi riformatori che hanno proposto, come spiegare perché le istituzioni accademiche e di ricerca non abbiano cercato di comprendere il ciclo di maturazione e consolidamento di alcune esperienze, o le loro frequenti trasformazioni in senso riduttivo e minimalista dovute ai cambiamenti politici o ai meccanismi di naturale "os-sificazione" (Giusti 1995) conseguenti alla coazione a ripetere o al mutare degli interessi e delle prospettive degli attori sociali coinvolti?

In questo contributo cercheremo di analizzare alcune trasformazioni che hanno segnato gli ultimi sei anni di vita (dal 2004 al 2009) di due esperienze partecipative metropolitane molto studiate dalla letteratura internazionale fino al 2003, ma ignorate nei loro sviluppi successivi: Porto Alegre e São Paolo. L'ambizione è leggere alcuni slittamenti di significato che tali mutamenti hanno sottinteso, specie per quanto riguarda il ruolo che la valorizzazione del conflitto svolgeva originariamente all'interno del meccanismo partecipativo istituzionalizzato. Il focus del saggio sarà centrato sullo specifico strumento del Bilancio Partecipativo (nonostante l'esistenza contemporanea di altre arene dibattimentali nelle due metropoli), e attinge a evidenze empiriche dedotte dall'analisi sul campo nelle due città ${ }^{5}$. L'ipotesi interpretativa che seguiremo si connette a proposte di lettura avanzate in altri numeri di questa rivista da autori interessati a leggere nel tempo le relazioni dinamiche tra le arene partecipative istituzionalizzate e l'organizzazione autonoma dei tessuti sociali. (www.ces.uc.pt/opp) e chiarisce come la coerenza di principi e modelli partecipativi in alcuni territori resista nel tempo, mantenendo una validità assoluta che li rende emulabili indipendentemente da fenomeni congiunturali che hanno portato alla loro soppressione.

5 Useremo dati emersi da ricognizioni qualitative sul campo; in particolare, dall'osservazione partecipante di alcuni processi partecipativi a São Paolo tra il 2001 e il 2009. I riferimenti ad altre esperienze di BP rappresentano "mirror-cases" riferiti a una bibliografia esistente. 


\section{Peculiarità del bilancio partecipativo e dei due contesti scelti}

Il Bilancio Partecipativo (BP), secondo i principi-quadro inaugurati a Porto Alegre tra il 1989 e il 2004 e poi riadattati in altri contesti, punta alla demistificazione delle procedure di bilancio - ovunque altamente tecnicizzate - e della tesi secondo cui sarebbe impossibile mettere in discussione con gli abitanti temi di elevato grado di complessità come quelli relativi alla programmazione economico-finanziaria. A tal fine, i BP tendono a reggersi su un'articolata architettura di spazi d'incontro differenziati che connettono continuamente "narrazioni" e "cifre" (Allegretti 2003), in modo che l'assunzione di decisioni non sia il mero frutto di un corretto scambio di opinioni razionali, ma un'opportunità per recuperare la funzione positiva delle emozioni e delle "narrazioni di storie" (Polletta 2006). In tal senso, la formulazione del BP scelta dalle due città oggetto della nostra riflessione attinge a una matrice comune ben rappresentata dalla configurazione adottata nel 1989 a Porto Alegre, ossia a un metodologia che, nella costruzione delle sue radici e dei suoi riferimenti teorici, dichiara di aver fuso sincreticamente una miscellanea di fonti di ispirazione diversissime (Fedozzi 2000, 2001) che vanno da Castoriadis ai teorici della deliberazione basata su flussi orizzontali di comunicazione, l'argomentazione razionale e l'ascolto reciproco (Habermas 1984). Sebbene tale modello partecipativo (che, pur schizofrenicamente "sincretico" nelle fonti di ispirazione, risulta coerente nella sua articolazione tra obiettivi dichiarati ed effetti conseguiti) non abbia sempre portato a esiti uniformi in termini di qualità discorsiva e valorizzazione dei saperi dei partecipanti (Talpin 2007), certo l'interesse suscitato risale ai due fattori nodali che lo caratterizzano: il reale potere di codecisione lasciato ai partecipanti nei confronti delle scelte oggetto di definizione di priorità e l'architettura che connette vari tipi di spazi concettualmente diversi.

In particolare (dopo modifiche significative introdotte nel 1994) il modello portoalegrense ha dato forma a due percorsi paralleli di dibattito pubblico - uno a base tematica, l'altro di appartenenza ai 17 distretti territoriali in cui è organizzato il territorio comunale - dove si alternano momenti in cui la partecipazione è aperta a ogni abitante e altri riservati a rappresentanti nominati dalla popolazione dei vari quartieri, concepiti per permettere una discussione più approfondita con meno interlocutori, e mantenendo un vincolo di mandato imperativo che lega i delegati alle zone di provenienza. Infatti, le loro proposte devono passare al vaglio del resto degli abitanti che decidono d'intervenire volontariamente nel processo prima di poter essere accolte dal Comune. Spazi informali di attraversamento del terri- 
torio (come le "carovane" per visitare collettivamente i luoghi dove gli abitanti hanno presentato proposte di investimento) e momenti più formali come quelli dove si valutano criteri sociali e di giustizia distributiva per tenere in conto le esigenze dei gruppi sociali più vulnerabili e delle porzioni di territorio più emarginate - puntano a far sì che l'accento sulla valorizzazione degli attori individuali non riduca le decisioni alla sommatoria di preferenze di singoli individui-partecipanti. Ciò per evitare che l'azione collettiva si riduca a un aggregato di dati individuali e permettere alle azioni dei singoli di combinarsi in dinamiche collettive virtuose (Diani 2008), possibilmente volte alla produzione di beni pubblici.

L'esistenza di varie procedure per risolvere impasse determinate da assenza di condivisione tra i partecipanti su obiettivi e investimenti della collaborazione instaurata con l'amministrazione comunale mostra come il BP non ponga l'accento sul consenso, ma piuttosto sulla graduale canalizzazione dei conflitti cui, per statuto originario, dovrebbe contribuire l'esistenza di chiare tempistiche di decisione connesse alle normative relative alle procedure di bilancio, difformi nei vari paesi (Allegretti 2005). Così, l'invenzione politica che ha preso forma negli anni Novanta risulta definibile come un dispositivo "conflittuato" (Caponetto 2002), nella misura in cui parte dal conflitto, ne è intrinsecamente pervaso e lo riassorbe in sé con l'obiettivo di superarlo $^{6}$. Lo scopo di un simile modello di arena pubblica è di non nascondere, ma anzi di valorizzare, i conflitti (anche quelli sostanziali e non riconciliabili) espressi all'interno dello spazio politico cittadino, considerandoli come il rovescio della medaglia dell'ordine (Pizzorno 2008) e cercando di contribuire a rompere o reindirizzare alcune dicotomie tradizionali (individuo/società, egoismo/solidarietà, locale/globale, bottom up/top down, conflitto/consenso, sapere laico/sapere esperto, ecc.) costruendo una geometria variabile di spazi di dibattito pubblico. Questi ambiscono a motivare i cittadini a partecipare facendo appello a motivazioni di natura individuale, ma li aiutano poi a sciogliere e diluire queste ultime in arene collettive di confronto dove, passando per l'esplicitazione dei conflitti, sia possibile perseguire la costruzione di visioni condivise e plurali e la scoperta di beni e valori comuni.

Per quanto concerne le peculiarità locali dei contesti analizzati, va detto che Porto Alegre (1,5 milioni di abitanti, capitale dello Stato Rio Grande do Sul) e São Paolo (11 milioni di abitanti, capitale dello Stato omonimo)

In casi come Bergamo (2006-2009) il BP ha posto la "propositività implicita" del conflitto al centro, adottando un percorso sperimentale "a macchia di leopardo" che di anno in anno sceglieva luoghi e oggetti del dibattito in funzione dell'essere oggetto di conflitti forti che li rendevano più attrattivi e comunicativi. 
hanno entrambe svolto un forte ruolo immaginifico relativamente alla sperimentazione del $\mathrm{BP}$, dato che il dibattito pubblico si è esercitato su oggetti non percepiti dai cittadini come "deboli" o "sfocati" ma - al contrario "prioritari" e spesso "indispensabili" per migliorare la qualità della vita e la convivenza sociale, e su cui pertanto molti abitanti hanno mostrato un'elevata disponibilità a mobilitarsi.

La netta differenza tra i due contesti locali ha influito sul radicamento dell'esperienza del BP e sulla sua capacità di durare nel tempo. Porto Alegre, infatti, ha conosciuto una solida continuità amministrativa dal 1989 al 2004, affidata a una coalizione progressista saldamente coordinata dal Partito dei Lavoratori (PT) e che contava pochi alleati di minoranza. I sindaci Dutra, Genro, Pont e Verle hanno tutti garantito al BP un'ampia centralità, mettendo i propri gabinetti al servizio del coordinamento di un processo che poteva essere trasversale solo se ubicato a monte della divisione tra assessorati. São Paolo, al contrario, ha assistito all'alternarsi del PT (promotore del BP prima con la sindaca Erundina, 1989-1992, e poi con Marta Suplicy, 2001-2004) con forze conservatrici. Al di là della discontinuità della sperimentazione, comunque, nella maggiore metropoli brasiliana il BP non è mai stato una priorità condivisa dall'intera coalizione, contando sempre molti nemici anche all'interno del PT (Sanchez 2004) e restando una politica tra le altre, ma non in grado di coordinare trasversalmente assessorati diversi.

L'origine delle due esperienze, quella che della Porta (2008) chiama «determinante processuale», è anch'essa molto diversa. Infatti, mentre per Porto Alegre esistono vari studi (Menegat 1998; Fedozzi 2000) che documentano la filiazione del BP da un serrato dialogo tra movimenti urbani e rappresentanti politici del PT, a São Paolo la sperimentazione è sorta o risorta in base ad accordi politici interni a correnti e personalità diverse del PT, rivelando un' origine top-down connessa alle proposte di un ceto intellettuale legato strettamente al partito, che chiedeva un segnale positivo di buona volontà nei confronti di una maggiore inclusione politica dei gruppi sociali più vulnerabili. Indubbiamente, però, vi sono state convergenze tra le due città, perché São Paolo ha sempre guardato con attenzione al modello portoalegrense, ma anche perché, per entrambe, le elezioni dell'ottobre 2004 hanno segnato la fine dell'esperienza progressista legata al PT.

Cosicché, stabilendo come variabile indipendente l'esistenza di dinamiche partecipative formalmente aperte a tutti i cittadini nell'ambito di un contesto di graduale trasformazione politico-partitica, è possibile riconoscere una consonanza tra le due città, specie tra il 2001 e il 2004, quando entrambe le metropoli hanno moltiplicato contatti e scambi di esperienze, 
nell'ambito di un comune movimento che le ha viste protagoniste della prima stagione dei Forum Sociali altermondialisti.

Nel seguito, assumendo come variabili dipendenti del percorso sperimentale alcune determinanti individuate da della Porta per analizzare le differenti caratteristiche degli esperimenti deliberativi (2008), ci soffermeremo soprattutto su alcune specificità dei due casi nell'interazione tra meccanismi istituzionali che rendono possibile la valorizzazione del conflitto e dinamiche non-istituzionali relative ad attori dei tessuti sociali legati al processo. Proponendo come indicatori per comprendere queste variabili fattori quali l'apertura a nuovi soggetti politici, il carattere consultivo o decisionale del BP e il suo disegno istituzionale, cercheremo di capirne l'influenza nello slittamento di senso dei percorsi sperimentati finora, che ha portato a due esiti diversi ma complementari.

A Porto Alegre, infatti, il radicamento del BP nell'immaginario sociale e nelle politiche pubbliche ha determinato una continuità formale, ma la nuova amministrazione (un'ampia coalizione composta di 10 formazioni partitiche di scarsa affinità, dai conservatori ai gruppi trotzkisti ${ }^{7}$ ) lo ha indebolito favorendo graduali trasformazioni interne del BP e delle condizioni esterne di contesto. Così ne ha attenuato la centralità e gli aspetti d'innovazione più contundenti, rendendolo "elusivo", secondo l'efficace espressione di Pellizzoni (2008). A São Paolo, invece, nonostante la violenta frattura voluta nel 2005 dalla nuova amministrazione conservatrice ${ }^{8}$, alcuni gruppi di delegati popolari formatisi in seno al precedente quadriennio di sperimentazione del BP hanno unito le forze e creato una rete di resistenza per obbligare l'amministrazione a reintrodurre principi di trasparenza e condivisione delle scelte, facendo leva sulle leggi nazionali approvate nel frattempo dal Governo centrale per valorizzare molte sperimentazioni partecipative del quindicennio precedente.

La nostra ipotesi è che lo svuotamento degli spazi deliberativi nelle due esperienze partecipative avvenuto dal 2005 a oggi si è legato a una graduale riduzione del potenziale spazio conflittuale delle stesse che, nel caso di Porto Alegre, ha determinato un annichilimento di gran parte delle forze sociali che avevano contribuito a dar forma al BP, mentre a São Paolo (fors'anche per la brutalità dell'interruzione) ha condotto alla reazione di una piccola porzione di tessuti sociali, organizzatisi affinché un quadriennio di sperimentazione coronato da importanti risultati (Campos et al. 2008) non fosse

7 La coalizione era presieduta dal sindaco-cantante Fogaça, ritiratosi a fine del 2009 per concorrere alla carica di Governatore.

8 Guidava la coalizione il Partido da Social Democracia Brasileira (PSDB) e - dal 2008 - il Partido Democrata (DEM). 
seppellito senza lasciare tracce nelle politiche del nuovo esecutivo. Nell' analizzare questo percorso differenziato non va dimenticato che all'origine dell'invenzione di molti dispositivi di gestione partecipativa in Brasile vi sono tre fenomeni convergenti e complementari: da un lato la "scoperta" della democrazia in senso lato e il bisogno di restituire un senso alle istituzioni locali (con la fine del ventennio della dittatura iniziata nel 1964); dall'altro l'incrudimento delle politiche neoliberiste e, infine, la necessità di riequilibrare un sistema elettorale binario imperfetto che, eleggendo con metodologie diverse esecutivi e assemblee elettive, determina spesso anomale convivenze che abbassano il livello di governabilità dell'ente pubblico'.

\section{Un panorama in trasformazione}

Parlando dell'impatto della de-simbolizzazione operata dal neoliberismo legata alla mercantilizzazione di oggetti e pratiche di socialità, Dufour (2005) ha sottolineato il farsi avanti di una «nuova forma filosofica della costruzione di un soggetto finora inedito» che prende forma «a partire dall'adattamento alla merce, promossa a unica realtà». Tale prospettiva aiuta a comprendere vari effetti del capitalismo attuale, operante non tanto per mezzo dei disposi-tivi di repressione istituzionale tradizionale (o di «coercizione e rieducazio-ne», ibidem), quanto piuttosto attraverso una "de-istituzionalizzazione", una deregulation applicata alle istituzioni politiche e sociali.

A cavallo tra gli anni Ottanta e Novanta (e almeno fino all'inizio del millennio), molti esperimenti partecipativi brasiliani hanno cercato di avanzare in direzione contraria, tentando di riscattare la dimensione simbolica degli esperimenti politici e rivalutando l'idea della "collettività" e della costruzione di un "senso in comune" attraverso un'articolazione discorsiva complessa tra azione collettiva e appartenenza territoriale. Fino al 2004, i BP di Porto Alegre e São Paolo sono stati forgiati come spazi di alto valore simbolico, raggiunto affiancando numeri e narrazioni in una sorta di ricostruzione semantica delle scelte pubbliche. Riconoscere tali caratteri controcorrente e il loro progressivo radicamento socio-territoriale non nega le contraddizioni che hanno segnato tali processi (Allegretti 2005; Campos et al. 2008), né afferma che i momenti assembleari abbiano rappresentato spazi liberi da gerarchie, manipolazioni e/o diatribe personali. Del resto, è inne- 
gabile che il conflitto fosse componente nodale, esplicita e pervasiva di tale concezione, come notato dalla migliore letteratura internazionale (Abers 2000; Baiocchi 2005; Wampler 2007), e in particolare nella definizione di countervailing power coniata da Fung e Wright (2003) e applicata al caso specifico di Porto Alegre.

La nozione - traducibile come "contro-potere cooperativo" e riferita a un potere sociale "di fatto" che non ha a che vedere con la divisione istituzionale dei poteri (Sintomer e Allegretti 2009) ${ }^{10}$ - aiuta a riconoscere l'esistenza di uno spazio costruito per stimolare la risoluzione cooperativa dei conflitti, piuttosto che in una prospettiva puramente agonistica di contro-poteri. Ma, soprattutto, evidenzia una componente nodale del percorso partecipativo strutturata intorno all'obiettivo di garantire il graduale empowerment dei gruppi sociali più fragili, al punto che alcuni autori (Baierle 2006) hanno parlato dell'emergere di una vera e propria "sfera pubblica delle classi subalterne" (espace public plébéien $)^{11}$.

A São Paolo e Porto Alegre (specie tra il 2001 e il 2004), si è compreso come l'apertura di spazi "conflittuati" non possa non tener conto della disuguaglianza esistente nell' accesso alle informazioni, così come nella molteplicità dei saperi necessari per digerirle e rielaborarle. Ignorando questi aspetti, infatti, si rischiava di generare percorsi partecipativi che riproducono le disuguaglianze sociali senza tentare di riequilibrarle agendo sui fattori che determinano il grado di potere, autorità e influenza che i vari individui (e i loro raggruppamenti) esercitano sulla configurazione delle scelte pubbliche ${ }^{12}$.

10 Il neologismo si differenzia dal "contro-potere" (counter-power), perché mette «simultaneamente l'accento su due dimensioni: una reale autonomia della società civile rispetto ai rappresentanti istituzionali e [...] una serie di meccanismi capaci di indebolire, o di neutralizzare, il potere e le prerogative politiche degli attori sociali normalmente dominanti» (Sintomer e Allegretti 2009). In Italia, la Rete del Nuovo Municipio ha coniato il termine "contro-municipio" per indicare varie pratiche che si propongono di costruire un "nuovo municipio" a partire da forme cooperative interne alla società.

11 «L'espressione salda derivazioni gramsciane e habermasiane. Si differenzia dalla nozione più ampia di sfera pubblica dell'Illuminismo [...] poiché si lega a movimenti d'emancipazione di classi subalterne e a lotte sociali di grande ampiezza. La questione sociale ne occupa una parte importante, e la composizione sociologica tende a favorire modalità espressive e di discussione che si allontanano [da quelle] brillanti dei salotti» (Sintomer e Allegretti 2009).

12 In Spagna città come Cordova hanno affrontato un patente conflitto tra organizzazioni sociali tradizionali (comitati di quartiere e associazioni di vicinato che reclamavano il rispetto del loro valore aggiunto in termini di saperi aggregati e di capacità rappresentativa dei bisogni del territorio) e i modelli di BP di derivazione sudamericana che valorizzano il cittadino-individuo indipendentemente dal suo grado di militanza associativa (Ganuza 2008). Ne sono nati modelli di BP "ibridi" con momenti dedicati alla cittadinanza organizza- 
Alcune trasformazioni avvenute nel campo discorsivo della partecipazione a partire dal mutamento amministrativo che ha segnato le due città a fine 2004 sono sintomo dello slittamento di significato che ci siamo proposti di analizzare. In particolare, si è andata diffondendo una prospettiva di lettura dei percorsi partecipativi imperniata sulla buona gestione dei partenariati a discapito della centralità del conflitto e della radicalizzazione democratica, in linea con modalità specifiche della socialità mercantile-individualista espres-sa da molti componenti delle nuove coalizioni di governo.

Per comprendere meglio queste nuove forme si potrebbe far riferimento alle nozioni antinomiche di «partecipazione rituale», «professionale» e «d'eccedenza» utilizzate da Pizzorno $(2008)^{13}$ o potremmo suggerire nuovi tentativi definitori che aiutino a chiarire i concetti per approssimazioni successive. Si potrebbe parlare, ad esempio, di partecipazionismo corporativo e partenariale entrambi collegati a una stessa tipologia processuale, ma in grado di ripercuotersi in maniera diversa sulla creazione di quello che habermasianamente può definirsi lo "spazio pubblico" e sulle nuove forme di lavoro e accumulazione.

Se consideriamo il partecipazionismo corporativo come modello riferito alle nuove strategie di gestione d'impresa (che coinvolga la soggettività dei funzionari/collaboratori nei processi produttivi) e quello partenariale come imperniato sulle nuove sinergie tra gestione istituzionale e terzo settore (privato sociale, ONG, cooperative, ecc.), la riformulazione dei BP di Porto Alegre e São Paolo pare cedere il passo a modelli partecipativi che rileggono le precedenti sperimentazioni di democrazia partecipativa nell'ottica del partenariato, sostituendole gradualmente con un orizzonte di democrazia associativa che, in senso tocquevilliano, appare come un modello di democrazia «tanto più forte quanto in essa cresce il peso della società civile organizzata» (Sintomer e Allegretti 2009).

Lo spazio aperto da dispositivi ispirati a questa logica tende, infatti, a favorire la partecipazione attiva di attori politico-sociali istituzionalizzati e formalizzati, soprattutto ONG, associazioni e altri attori collettivi (sindacati, forze partitiche, ordini professionali, ecc.) dotati di una "storia", che ap-

ta e altri estesi a chi si presenta a titolo individuale.

13 Il primo tipo includerebbe i fenomeni partecipativi ritualizzati (ripetuti nel tempo) e le manifestazioni che esprimono appartenenza a un gruppo politico/sociale (dove il rito serve a rafforzare e riprodurre la solidarietà di gruppo e il riconoscimento reciproco). La partecipazione professionale sarebbe strumentale, e include anche burocrazia, gruppi di interesse e opinion-maker. La partecipazione di eccedenza riguarda i movimenti sociali, ed eccede i confini della razionalità strumentale e dell'agire calcolatore, irrompendo sulla scena quando altri canali di partecipazione entrano in crisi e declinano, erodendo la loro capacità di generare entusiasmo. 
paiono sovente come legittimatori di decisioni negoziali maturate nelle tradizionali istanze decisionali della democrazia rappresentativa. In queste situazioni, non è infrequente uno slittamento dal concetto di conflitto a quello di competizione (per il finanziamento di progetti, appalti, gestione di servizi in outsourcing, ecc.), tipico di una concezione della sussidiarietà orizzontale subalterna rispetto all'importanza data all'accezione verticale dello stesso principio. Come se il rapporto tra istituzioni politiche e società civile fosse solo un corollario dell'importante riordino delle relazioni tra i diversi istituti di governo propri della democrazia rappresentativa.

Nell'ultimo quinquennio, la graduale riformulazione (Porto Alegre) o la sostituzione (São Paolo) del precedente modello di BP si è ispirata a un approccio partecipazionista del secondo tipo, che pare predominante anche nella riformulazione di altre esperienze brasiliane di BP che hanno avuto formale continuità, come Belo Horizonte (Sampaio 2010) o Santo Andrè.

Tale ridefinizione sottende il passaggio da una polarità amico-nemico (vicina ai termini usati da Schmidt per definire l'essenza della politica), a tipologie di rivalità tra concorrenti, dove sono soprattutto gli amici che sono chiamati a partecipare, pur all'interno di meccanismi non esclusivi, che lasciano formalmente aperta qualche porta alla presenza di voci dissonanti. Queste ultime, comunque, non paiono incoraggiate a farsi presenti e non sono poste al centro dei meccanismi di informazione e di accesso agli spazi di dialogo sociale previsti.

Se a Porto Alegre una parziale regolamentazione del BP resta ancora in piedi a tutela di una sua maggiore inclusività potenziale, a São Paolo la discontinuità introdotta con la formale cancellazione del BP ha determinato un'eclatante assenza di regolamenti che diano certezze sulla trasparenza dei trasferimenti di risorse pubbliche a soggetti associati che operano nella sfera socio-economica, rafforzando l'idea di storni frutto di logiche negoziali collegate al malcostume politico di matrice clientelare ${ }^{14}$.

14 Molti BP europei, africani o asiatici cercano di ancorare lo sperimentalismo a strutture "solide" del panorama sociale preesistente, e così disperdono le novità di un meccanismo che generalmente predilige (specie in Brasile) una «geometria variabile di aggregazioni di interessi che tendono a non essere permanenti, ma riconfigurano continuamente la loro geografia in base agli obiettivi via via raggiunti» (F. Frieri, Corso di Formazione EQUAL “Orçamento Participativo: mais participação, melhor democracia”, Coimbra 2526/09/2008). 


\section{La retrocessione del potenziale di valorizzazione del conflitto}

Nel seguito, è su quattro variabili dipendenti legate al disegno istituzionale del BP e alla sua collocazione all'interno dell'organigramma politicoamministrativo che ci soffermeremo, valorizzando il loro peso di determinanti (della Porta 2008) che, interagendo con altre modificazioni legate alle capacità e alle modalità di azione collettiva degli attori partecipanti, possono determinare un circolo vizioso nella dissoluzione dell'identità originaria del processo partecipativo. L'ipotesi è che il disegno istituzionale abbia avuto un peso significativo nella riformulazione delle politiche partecipative di São Paolo e Porto Alegre, uscendo dall'ottica della valorizzazione esplicita del conflitto sulla strutturazione delle politiche pubbliche come "materia prima" cui attingere per costruire progetti condivisi e identificare collettivamente beni e valori da difendere.

\subsection{Ubicazione del centro nevralgico del processo partecipativo}

Nomi e immagini legate ai processi partecipativi rivestono forte valore simbolico nel fotografare i mutamenti della loro dimensione sostanziale e metodologica. A São Paolo, nel periodo 2001-2004, l'organismo comunale responsabile d'incentivare e regolare la partecipazione degli abitanti si chiamava Coordinamento del BP, e dipendeva da un assessorato di snodo, delegato al coordinamento politico tra le diverse componenti dell'amministrazione. Sebbene, a causa della relazione tra le correnti interne al PT, vari limiti e ostacoli avessero compresso la messa in opera del BP, il processo partecipativo era supportato da un'articolazione complessa e plurale, dato che esisteva anche il Coordinamento della Partecipazione Popolare, responsabile dell'interfaccia con gli altri Consigli e Consulte tematiche municipali (Consiglio degli Anziani, dei Giovani e degli Adolescenti, Coordinamento delle Donne, ecc.). Con il cambio di leadership politica del gennaio 2005, il Coordinamento del BP ha subito uno svuotamento politico, metodologico e funzionale $^{15}$. Da allora, il nome dell'organismo è stato alterato varie volte, divenendo Coordinamento della Partecipazione Sociale (legato all'Assessorato alla Partecipazione e ai Partenariati creato nel 2005), e oggi Coordinamento della Convivenza, Partecipazione e Imprenditoria Sociale, che, fin dal nome, evidenzia il nuovo senso dell'organismo nell' ambito di una con-

\footnotetext{
15 La nuova Giunta inviò una lettera aperta al coordinamento del Programma URBAL (Rete n. 9 sul BP) per rifiutare ogni futuro impegno, con duri attacchi al concetto di BP.
} 
cezione partenariale della partecipazione. A Porto Alegre, il passaggio è stato meno traumatico, dato che il BP non è stato mai cancellato, anzi, essendo nodo della contesa politica tra i candidati dei maggiori schieramenti (il PT e la variegata coalizione vincitrice costituitasi contro di lui), permane visibile come percorso autonomo, e mantiene formalmente un notevole risalto, fin dalla pagina web del Comune. Qui, la nuova "ubicazione" del vecchio percorso partecipativo dentro un nuovo contenitore istituzionale, l'Assessorato al Coordinamento Politico e alla Governance Locale (separato dall'Assessorato alla Gestione Strategica e dal Gabinetto di Programmazione Finanziaria), evidenzia uno slittamento di significato del percorso stesso.

Nei due casi, non si tratta d'una trasformazione meramente nominale di alcuni organismi comunali ma di una frammentazione e di una marginalizzazione politica e funzionale, che sottende una nuova logica di comportamento nella costruzione delle relazioni con i diversi attori sociali.

\subsection{Apertura del processo ad attori diversificati}

L'inclusione nei processi di BP di attori politici differenziati, specie se hanno tradizionalmente difficoltà ad accedere ai canali formali della partecipazione, è il primo fattore che condiziona la presenza o l'assenza di conflitto come principio plasmante dei processi. Evidentemente, un percorso che apra spazi di partecipazione a persone che vivono in quartieri residenziali degradati, o a senzatetto, può generare un potenziale conflittuale maggiore rispetto al mero coinvolgimento di abitanti di zone benestanti o (tema pertinente nel contesto brasiliano) di compound separati dal resto del tessuto abitato.

Se in alcuni processi europei di BP gli abitanti proprietari di abitazioni hanno maggiori motivazioni a prendere parte attiva alla trasformazione della città per valorizzare i propri beni (D’ Albergo e Moini 2005), i casi brasiliani mostrano come anche il solo possesso di una baracca riesca a motivare potentemente gruppi sociali tradizionalmente esclusi.In una megalopoli come São Paolo, con un quadro di molteplici forme di segregazione, l'adozione di meccanismi partecipativi deve preoccuparsi di equalizzare l'accesso ai canali di deliberazione pubblica. Così, a partire dal 2001, il BP ha creato azioni affermative nei confronti dei soggetti sociali vulnerabili, stabilendo criteri differenziali per coinvolgere alcuni gruppi sociali nelle assemblee. La politica di discriminazione positiva ha avuto come target donne, cittadini afro-discendenti, indigeni, giovani, collettivi di persone a differen- 
te orientamento sessuale, soggetti diversamente abili e senza fissa dimora. Chi si fose autodichiarato appartenente a questi gruppi (con un'assunzione pubblica di responsabilità), poteva contare sull'elezione di delegati e consiglieri popolari secondo criteri di proporzionalità inferiori a quelli necessari per eleggere delegati nelle assemblee territoriali ${ }^{16}$. In tal modo (non senza conflitti patenti, anche relativamente ai criteri adottati) gli appartenenti a tali gruppi avevano maggiori possibilità di realizzare investimenti pubblici a loro beneficio. L'apertura alla canalizzazione di nuove esigenze sociali specifiche nel processo di BP ha moltiplicato i fuochi del conflitto nella sfera pubblica (su questioni di genere, etnia, età, ecc.), ma ha permesso anche l'emergere di scelte solidali e di meccanismi di evidenziazione di squilibri socio-economici o culturali che fino ad allora non riuscivano a rendersi espliciti nella loro gravità (Sanchez 2004).

Oggi, a São Paolo non vi è più traccia di simili criteri nei percorsi partecipativi sporadici che hanno soppiantato il BP nella discussione, ormai a carattere solo consultivo, di questioni economico-finanziarie. La stessa divulgazione delle assemblee partecipative è ristretta, come rivelano l'analisi del profilo dei partecipanti e un'inchiesta sui cittadini non-partecipanti (Siqueira e Campos 2009). Al di là dell'assenza di preoccupazioni esplicite sul coinvolgimento di gruppi sociali deboli, si rileva un aumento proporzionale della partecipazione di organismi legati al mondo delle imprese, del commercio, della pubblica sicurezza, dei mass-media di area conservatrice, di enti come il Rotary Club e i Lions. Nella stessa misura, si nota un aumento nella partecipazione di consulenti e collaboratori di assessori e consiglieri regionali e comunali ${ }^{17}$. Rispetto ai processi del passato, centrati sul coinvolgimento di cittadini-individui, cresce la presenza di attori politici a carattere collettivo. L'apertura dei nuovi spazi partecipativi che hanno soppiantato il BP diviene selettiva, favorendo, già dalle fasi informative ${ }^{18}$, la comunicazione con settori specifici della società civile organizzata di classe

16 Ad esempio, se erano richiesti 15 residenti per eleggere un delegato in rappresentanza della zona, ne bastavano 5 o 2 per un rappresentante dei presenti che si autodichiaravano membri di comunità indigene o gruppi LGBT.

17 F. Siqueira ha seguito tutti gli incontri pubblici sui temi del bilancio, sia nel periodo 2001-2004 che dopo. Il profilo dei partecipanti è ricavato dagli atti.

18 Le nuove strategie di divulgazione delle riunioni spiegano in parte la scomparsa di alcuni settori sociali. L'uso maggiore di internet e le pubblicità su giornali e riviste - in precedenza si usavano auto con megafoni nelle strade - può portare ad una progressiva elitarizza-zione del percorso, in una città polarizzata con grandi sacche di miseria. In altri casi in cui il BP considera centrale l'uso delle piattaforme web (come a Lisbona o a Belo Horizonte) si usano strumenti (ad es. bus equipaggiati di computer) per sostenere i cittadini meno alfabetiz-zati sull'uso delle TICs. Questo motodo di informazione non viene utilizzato a São Paolo. 
media e con forte potere d'acquisto. Tale variazione del pubblico ha contribuito alla riduzione della dimensione conflittuale delle esperienze, aumentando la concorrenza tra diversi attori, e ciò in parallelo alla sottrazione del potere decisionale delle assemblee pubbliche.

Anche in termini quantitativi, la differenza di partecipazione nei due modelli di São Paolo è ben visibile. La media di 500 assemblee e riunioni partecipative annuali raggiunta tra il 2001 e il 2004 (che includevano divisioni transcalari del territorio: circoscrizioni decentrate, frazioni, zone censuarie) cercava di garantire un'attenzione specifica per i territori abbandonati, mentre i numeri della partecipazione mostravano una crescita progressiva: dai 33.000 partecipanti del 2001 agli 80.000 del 2003. Dal 2005, la media delle riunioni sul bilancio pubblico è andata riducendosi drasticamente, e il dato risulta aggravato dalla cancellazione dei percorsi di formazione su un tema non semplice come quello del bilancio. Nel 2005, sono state realizzate 37 plenarie; nel 2006 e 2007 solo 5 riunioni, e nel 2008 nessun incontro pubblico nella fase di elaborazione del bilancio municipale. $\mathrm{La}$ violazione della Legge di Responsabilità Fiscale Federale ${ }^{19}$ ha generato una doppia denuncia giudiziaria da parte dei movimenti sociali paulisti, indirizzata al Ministero Pubblico Federale e a quello Statale (Promotoria da Cidadania), lo stesso che nel 2010 è riuscito a far annullare il Piano Regolatore cittadino per assenza di partecipazione nella sua stesura.

$\mathrm{Nel}$ caso di Porto Alegre, piuttosto che una riduzione nel numero degli incontri e dei presenti, si rileva la mutazione delle logiche di auto-reclutamento. Infatti, la modifica del Regolamento Interno del BP nel 2008 ha permesso che i delegati popolari siano rieleggibili da un anno all'altro, lasciando spazio alla costruzione di potentati locali che si danno da fare per riempire con cittadini a loro favorevoli le riunioni del primo ciclo annuale, dove si votano i delegati che accompagneranno e analizzeranno nei dettagli le proposte di bilancio nel tempo. Qui il conflitto si è accresciuto, ma solo nella prima parte del ciclo annuale, in una logica competitiva che punta a stabilizzare il potere di alcune guide che poi concerteranno con altre la lista delle priorità da proporre al finanziamento pubblico.

Tale logica converge con quella dell'amministrazione portoalegrense, che dal 2005 ha dato centralità al processo di Governança Solidaria, un concetto dai contorni nebulosi che, alla luce dei percorsi che ne fanno parte, sembra centrato su due pilastri (Busatto 2005): dare priorità ad accordi parteneriali tra istituzioni locali e settori organizzati della società (gruppi economici del privato sociale, cooperative o ONG con base nelle favelas) e 
spalmare la partecipazione oltre la fase della co-decisione, per estendere gli accordi alle fasi di gestione di opere e servizi pubblici. In tale ottica, trova coerenza la previsione dei cosiddetti emendamenti, un pacchetto di oltre 170.000 euro attribuito ai consiglieri popolari di ognuno dei 17 distretti di BP della città, su cui gli stessi hanno libertà quasi totale di decisione. L'offerta di discrezionalità decisionale su tali fondi ha rafforzato la competizione nella fase di elezione dei delegati del ciclo di BP, creando uno zoccolo duro di delegati e consiglieri permanenti legati al Comune da relazioni clientelari che rischiano di riportare il dispositivo partecipativo indietro di venti anni.

Sebbene manchino dati precisi a supporto di questa trasformazione sociologica del pubblico più attivo del $\mathrm{BP}$ di Porto Alegre (solo nel 2011 l'Osservatorio di Porto Alegre pubblicherà le conclusioni della sua inchiesta sui partecipanti al BP, di cui alcuni risultati sono anticipati in Fedozzi e OBSERVAPOA, 2009) per chi svolge osservazione partecipante, come l'ONG "Cidade", la gentrificazione in atto pare evidente. L'insieme delle trasformazioni che racchiudono il BP in un nuovo quadro d'insieme ha trasformato la sua relazione con i concetti di conflitto e consenso, privilegiando una lettura partenariale della partecipazione che mette in sordina le tensioni, convogliandole verso canali d'espressione esterni alle arene istituzionalizzate.

\subsection{L' architettura degli spazi per partecipare}

Tra i criteri utili a comprendere l'apertura di un dispositivo partecipativo all'espressione e alla valorizzazione dei conflitti vi è l'esistenza di spazi (permanenti o puntuali) di negoziazione che favoriscano la manifestazione d'interessi antagonisti, e forniscano mezzi isonomici per la graduale risoluzione del conflitto.

Tradizionalmente, nelle assemblee non si esprime la maggior dinamica conflittuale. Il loro obiettivo è raccogliere i più variegati generi di proposte, che nelle tappe successive saranno selezionate e gerarchizzate in base anche alla fattibilità tecnico-giuridica, la quale rappresenta uno spazio di discrezionalità per i tecnici municipali, foriero di conflitti sul merito delle soluzioni con gli abitanti. Nei BP, i maggiori conflitti si sviluppano nelle istanze deliberative, arene del processo dove si stabiliscono priorità rispetto a liste più ampie di bisogni e desideri espressi dalla popolazione. Specie 
nelle grandi città, si tratta di organismi come i Forum dei Delegati ${ }^{20}$ e i Consigli Popolari del Bilancio Partecipativo (COP), istanza massima a carattere decisionale di molte sperimentazioni. Il conflitto instaurato nei COP in funzione delle scelte sulla distribuzione delle risorse non ha mai impedito che risultasse visibile una tendenza redistributiva delle decisioni finali rispetto alle risorse allocate sul territorio per mezzo delle decisioni degli abitanti (Campos et al. 2008).

Così, in casi come São Paolo, l'attuale assenza di attenzione per la costruzione di un' architettura istituzionale che contempli simili spazi deliberativi/decisionali indebolisce significativamente la dimensione conflittuale e quella solidale del processo. Nella capitale paulista, dal 2005, la deregolamentazione dei meccanismi partecipativi ha visto cessare anche la pubblicazione degli atti delle riunioni pubbliche, che in precedenza rappresentavano punti nodali nel processo ${ }^{21}$. Nel mandato 2005-2008, i movimenti sociali e persino il Consiglio Comunale hanno promosso vari tentativi di ri-regolamentazione della partecipazione. A tutti è stato messo il veto dalla Giunta, anche dopo l'approvazione ufficiale in Consiglio $^{22}$.

Nel caso di Porto Alegre, dove l'autoregolamentazione del BP continua a funzionare, non vi è stato l'annullamento delle istanze decisionali, ma, come mostrano i numeri 21/26 della rivista De Olho no Orçamento, la tradizionale e costruttiva conflittualità interna del COP è stata narcotizzata per sfinimento, sia riducendo i margini decisionali a fette via via più ridotte del bilancio, sia rarefacendo le interazioni tra Giunta e COP attraverso una strategia del silenzio che riduce il numero delle risposte istituzionali date su temi come la differenza tra le cifre stanziate nel bilancio e i costi reali delle opere (bilancio a consuntivo), il cui aumento sovente incide sulla realizzabilità di altri investimenti, specie laddove vi siano limiti invalicabili alla spesa generale. La previsione degli emendamenti su cui possono decidere liberamente $\mathrm{i}$ consiglieri delle varie zone ha abbassato la conflittualità all'interno del COP, dotando i vari rappresentanti popolari di margini diversi di negoziazione che non li obbligano a rinunciare per forza a scelte di loro peculiare interesse. La lettura degli ultimi Piani degli Investimenti mostra come questi emendamenti vanno a favore soprattutto di asili privati, centri di formazione professionale e altre istituzioni di quartiere i cui gestori, come è già stato osservato da Fedozzi (2007) finiscono per costituire lo zoccolo duro dei consiglieri popolari del COP. Tale situazione si è aggrava-

\footnotetext{
20 Istanza permanente del BP che unisce rappresentanti territoriali, tematici e dei vari gruppi sociali eletti nelle assemblee.

21 Per dettagli, vedi Diário Oficial do Município 2001-2004.

22 Cfr. Progetto di Legge n. 108/2005.
} 
ta nel momento in cui altre istanze partecipative - come il Consiglio Municipale di Sviluppo Urbano e Ambientale (CMDUA), che vigila sul rispetto e la modifica degli strumenti urbanistici - hanno visto sostituire i rappresentanti di alcuni movimenti conflittuali con militanti delle organizzazioni edili, inclusi muratori i cui interessi sulle ipotesi di sviluppo edilizio illimitato difficilmente confliggono con quelle dei costruttori (o speculatori) che danno loro lavoro.

Un'ultima questione legata all'architettura dei processi è quella della scelta dei luoghi dove svolgere i dibattiti. A São Paolo, nel 2001-2004, le riunioni del $\mathrm{BP}$ erano realizzate nei 96 distretti comunali, con più di una riunione per distretto, senza contare quelle autorganizzate nei vari quartieri per preparare le strategie degli abitanti. Il modello del 2005-2010 riduce drasticamente il numero delle riunioni, ma, soprattutto, cambia l'ottica di scelta dei locali che le ospitano. Non più scuole pubbliche, teatri, biblioteche, sedi d'associazioni di quartiere, palestre, campi sportivi e scuole di samba; piuttosto auditorium di Università private, associazioni militari e sedi delle Subprefeituras (Consigli Circoscrizionali decentrati, a São Paolo non elettivi). Luoghi spesso lontani dai grandi agglomerati urbani più poveri dove, nella maggioranza dei casi, neppure giungono le convocazioni e gli inviti a partecipare. A Porto Alegre la situazione è meno drammatica, sia per il numero che per la localizzazione territoriale delle assemblee. Eppure si percepisce un crescente mutamento della tipologia di spazi. In zone come Anchieta o Gloria, i collegi dei Preti Maristi e gli istituti religiosi parificati divengono importanti riferimenti territoriali, fino a essere identificati dal pubblico con il processo. Dato che i luoghi non sono mai neutri ma possiedono una carica comunicativa implicita, come ben provato dal BP di Roma XI (Sintomer e Allegretti 2009), una trasformazione degli spazi può avere effetti «attraenti per gli amici e respingenti per i critici o i partecipati non allineati», cioè contribuire a una selezione darwiniana del pubblico del BP.

\subsection{Grado di empowerment del processo}

Il ruolo specifico che un'arena deliberativa occupa nel policy-making (ossia il suo essere uno spazio meramente consultativo/argomentativo o piuttosto - argomentativo/decisionale) è non solo una determinante importante del grado di empowerment che il processo permette (della Porta 2008), ma anche un indicatore per leggere il potenziale conflittuale delle esperienze. Di solito, in Brasile, i processi a carattere decisionale producono dinamiche di encaminhamento propositivo (suggerimenti in forma pro- 
attiva per l'avvio di decisioni da implementare in tempi rapidi), anche se ciò, purtroppo, non garantisce di per sé che le richieste siano poi prese in carico e realizzate dalle istituzioni (come si nota in quasi tutte le esperienze di BP, che accumulano "arretrati" tra le realizzazioni previste). In ogni caso, il fatto che ai cittadini siano garantiti spazi per deliberare e stabilire priorità tra le proprie proposte genera effetti di tensione nell'apparato istituzionale, quando questo si trova davanti alla decisione di accogliere o rigettare le proposte e motivare la scelta in maniera convincente, per poi dare concretezza alla realizzazione.

Per lo più i BP (in particolar modo in Brasile) si fondano su un impegno delle amministrazioni municipali a incorporare un certo numero di richieste presentate dai cittadini a scale diverse (quartiere/distretto/intera città) e su ambiti tematici prestabiliti. I processi consultivi, ovviamente, non hanno questo obbligo. Oggi, in Europa, stanno divenendo frequenti i BP basati su un ascolto selettivo dei bisogni dei cittadini da parte delle istituzioni elettive (Sintomer e Allegretti 2009): in questi casi gran parte del potenziale innovatore del modello brasiliano a carattere decisionale va disperso.

Le due città presentano una differenza sostanziale nel grado di continuità dato all'originario modello in vigore all'inizio del decennio. A Porto Alegre, infatti, il BP ha mantenuto formalmente il carattere di partecipazione piena (Pateman 1970) con esiti visibili (King et al. 1998), dove i partecipanti assumono, sulla base d'un regolamento guida che contribuiscono annualmente a emendare, varie decisioni che l'Amministrazione si impegna a sottoscrivere. Si è però ridotto il grado d'incidenza, ossia la percentuale delle scelte sottoposte a decisione pubblica ${ }^{23}$. A São Paolo, invece, la formale soppressione del BP, ancorché parzialmente reintegrato da nuove riunioni pubbliche legate agli obblighi della Legge di Responsabilità Fiscale, ha portato a una formale retrocessione da un modello di controllo a uno di mera consultazione (Bishop e Davis 2002). Una delle principali innovazioni introdotte dal BP tra il 2001 e il 2004 era stato il carattere deliberante del processo (e soprattutto del COP), che lo differenziava dal panorama delle molte consulte comunali già esistenti, che operavano come sorta di gruppi di raccolta di suggerimenti e opinioni che i gestori pubblici erano liberi di tenere in conto o ignorare a seconda dei giudizi di valore, delle proprie priorità, della congiuntura o dell'opportunità attribuite loro a proprio insindacabile giudizio ${ }^{24}$.

I dati diffusi dal Comune configgono con quelli di vari ricercatori indipendenti (Cidade 2007; Schneider e Baquero 2009; Muzell 2010; Langelier 2011) che sostengono che lo spazio di manovra del BP si è ridotto notevolmente, pur non concordando sulla percentuale. Quando funzionava il COP (2001-2004), la votazione finale sulle priorità da fi- 


\section{Una conclusione aperta}

Nel testo abbiamo evidenziato alcuni ambiti di riflessione utili a valutare i rischi di progressiva destrutturazione e de-simbolizzazione di processi partecipativi nati con l'obiettivo di sovvertire alcune logiche delle tradizionali forme di concertazione territoriale, ma che oggi vengono gradualmente rias-sorbiti in logiche simili. La nostra analisi ha toccato un periodo storico ne-gletto dalla letteratura (posteriore alle elezioni amministrative dell'ottobre 2004) in due città-simbolo dell'innovazione partecipativa degli inizi del mil-lennio. Nei due territori, sembra prendere forma quella che Dagnino (2004) definisce «una crisi discorsiva» che attraversa molte esperienze di costruzio-ne democratica e porta a una «convergenza perversa» tra il progetto neolibe-rista in America Latina e il progetto di democratizzazione partecipativa. Il mutamento dei modelli partecipativi si lega a visioni di forze politiche diver-se da quelle che avevano proposto le sperimentazioni originarie di BP, ma, soprattutto nel caso di Porto Alegre, dove la denominazione del processo è rimasta immutata, e la supposta continuità col passato pare formalmente sal-vaguardata, vi è il rischio che le nuove logiche contribuiscano a un mutamen-to diffuso dell'immaginario che può toccare anche altri $\mathrm{BP}$ che a esso vanno ispirandosi, contribuendo così $\mathrm{a}$ confonderne i principi strutturanti.

Per Fung e Wright (2003) l'articolazione tra contropotere cooperativo, conflitto e collaborazione partecipativa è vitale per dar forma a efficaci modelli di Empowered Participatory Governance, che rifuggano gli estremi del-la dominazione e della cooptazione. Secondo i due autori, se la transizione da dinamiche reattive di mera protesta a dinamiche collaborative deve affrontare molte barriere (la scala politica, le competenze organizzative, le differenze nella costruzione dei significati politici e delle fonti psicologiche di solidarietà), il grado minore o maggiore di countervailing power sarà determinante per definire gli esiti di un tale passaggio. Nei casi esaminati, le due fonti principali di contropotere cooperativo individuate da Fung e Wright (le mobilitazioni di gruppi e movimenti locali e il profilo dei governanti) sono state entrambe colpite dai cambiamenti politici dell'ottobre 2004. Il caso paulista, segnato dalla contestazione, persino giuridica, della legittimità delle preesistenti pratiche partecipative da parte del nuovo sindaco, rappre-senta un estremo di questa trasformazione; ma i dati presentati da Baiocchi (2005) e da Fedozzi (2007) in relazione a Porto Alegre mostrano, comun-

nanziare era la base per strutturare le politiche pubbliche e un valore-distintivo del BP, un DNA in contrasto con il complesso ed ermetico ciclo di bilancio tradizionale della maggiore città brasiliana. 
que, come la guida politica del progetto partecipativo e il suo disegno istituzionale svolgano un ruolo fondamentale nella valorizzazione del potenziale conflittuale e della vitalità del BP. Queste, infatti, impattano direttamente sia sulla capacità decisionale del processo, che su quella di ridurre le asimmetrie tra gli attori protagonisti degli spazi decisionali.

In un'epoca di crisi economico-finanziaria (spesso strumentalmente esibita per giustificare nuovi tagli alle politiche pubbliche) la ricerca di una pax sociale basata sulla cooperazione tra attori diviene obiettivo primario di molti processi partecipativi, venendo considerata come pre-condizione per rendere un territorio più competitivo nel mercato globale. Ciò pare condurre a nuove forme istituzionali, preoccupate soprattutto di contribuire alla governance degli stakeholders, che rimettono l'accento sulla centralità di negoziazioni tra corpi sociali intermedi legati alla logica rappresentativa come enti, associazioni e ONG dai profili più svariati con cui lo Stato costruisce rapporti partenariali di reciproco interesse, non necessariamente finalizzati a interessi comuni. La logica degli stakeholders organizzati in perenne negoziazione (quelli che, per il Sud del mondo, Villamán definisce «società civile profumata») sembra re-invadere anche il campo di percorsi come i BP, annacquandone le peculiarità e disperdendone la capacità d'innovazione. Un mainstream di riflusso va così gradualmente sostituendo alcune dinamiche politiche nate per favorire il posizionarsi del conflitto nello spazio pubblico e aprire arene di espressione alle richieste di voci non-organizzate e di attori sociali eccentrici.

Come osserva Villamán (2010), una simile trasformazione rafforza la contrapposizione tra un modello di democrazia e di cittadinanza a carattere politico/formale e uno a carattere sociale dove il processo partecipativo non è più un "ponte" tra le due famiglie, ma un soggetto in causa aggregato a una concezione del primo tipo. Non va, infatti, dimenticato che oggi le esperienze di democrazia partecipativa «si danno in un quadro di ritorno allo Stato» differente da quello dei due decenni precedenti; e pertanto non basta che «la società civile si distanzi dal mercato, ma è indispensabile che costruisca un punto di vista proprio rispetto allo Stato [...], una proposta alternativa all'ordine sociale vigente marcato dall'ingiustizia» (ibidem). Ciò, nella visione del sociologo dominicano presidente del Consiglio Nazionale per la Riforma dello Stato ${ }^{25}$, non solo obbliga «a relativizzare la cultura del consenso come obiettivo della partecipazione, e a vedere quest'ultima come spazio per l'espressione di dissensi e la costruzione di visioni convergenti alla statuizione per legge del BP (2007) e al suo inserimento in Costituzione (gennaio 2010). 
che riconoscano la diversità», ma soprattutto richiede che la società civile «non si accontenti di misure di trasparenza e rendicontazione, che evitano gli inganni ma non risolvono l'ingiustizia sociale» e imperni la sua battaglia sul ritorno a percorsi partecipativi «centrati sulla necessità di costruire giustizia distributiva e inclusione sociale» (ibidem).

Un simile monito pare adattissimo al caso del processo di BP di Porto Alegre, dove, adattando le parole di Villamán, potremmo dire che il processo partecipativo non rischia di diventare escludente per assenza di presenza popolare (dato che ancora raduna molti partecipanti dalle aree più bisognose del territorio), ma piuttosto per l'assenza di un progetto e di un punto di vista popolare che sia antagonista alla visione addomesticata di partecipazione che pare veicolare l'amministrazione comunale. Guardando a queste vicende dall'Europa, rischi e sfide emergenti dal mutamento di significato rilevato nelle due città brasiliane paiono ancora più estremizzati, dato che il modello dei nostri BP (nonostante il riferimento di prammatica al modello ispiratore di Porto Alegre) giunge spesso già geneticamente modificato. Ossia dà forma a creazioni moderatamente sperimentali, maturate "dall'alto" all'interno d'istituzioni pubbliche, e sovente legate a preesistenti organigrammi sociali e amministrativi di cui non sono interessati a sovvertire totalmente le logiche. Non è un caso che siano rari gli esempi di BP europei che mettano in evidenza elementi di giustizia distributiva o favoriscano l'empowerment e l'emancipazione di gruppi sociali tradizionalmente esclusi dalla negoziazione delle scelte di governo (Allegretti 2009b).

È perciò indispensabile lavorare a un futuro ampliamento del potenziale di autonomia delle esperienze partecipative, e, al contempo, a una revisione dell'orientamento a valorizzare ambiti di dialogo sociale non-conflittuale. Le due exit strategy dal vicolo cieco in cui alcuni processi partecipativi si stanno infilando dovranno essere condotte insieme. Altrimenti, vi è il rischio di portare avanti, attraverso percorsi debolmente partecipativi, una concezione non problematizzata delle politiche pubbliche che si appaga della verifica di conformità alle previsioni normative o ai vincoli economico-finanziari, piuttosto che leggerle come una forma di azione collettiva di cui l'autocontrollo sociale e la capacità di valorizzazione del conflitto sarebbero componente nodale.

\section{Riferimenti bibliografici}

Abers R. (2000), Inventing Local Democracy: Grassroots Politics in Brazil, London, Lynne Rienner. 
Allegretti G. (2003), L'insegnamento di Porto Alegre. Autoprogettualità come paradigma urbano, Firenze, Alinea.

Allegretti G. (2005), Porto Alegre: una biografia territoriale. Ricercando la qualità urbana a partire dal patrimonio sociale, Firenze, Firenze University Press.

Allegretti G. (2009a), "Porto Alegre: il mito va in pezzi”, Carta, 31.

Allegretti G. (2009b), "Giustizia sociale, inclusività e altre sfide aperte per il futuro dei processi partecipativi europei”, in Allegretti U. (a cura di), Democrazia Partecipativa. Esperienze e prospettive in Italia e in Europa, Firenze, Firenze University Press.

Avritzer L. (2006), Sociedade Civil e Participação Social no Brasil, Belo Horizonte, DCE/UFMG.

Baierle S. (2006), "Les ONG et l'insoutenable marchandisation de la solidarité", Mouvements, 47-48.

Baiocchi G. (2005), Militants and Citizens. The Politics of Participatory Democracy in Porto Alegre, Stanford, Stanford University Press.

Bishop P. e Davis G. (2002), "Mapping public participation in policy choice", Australian Journal of Public Administration, 61, 1, pp. 14-29.

Bobbio L. (2002), I governi locali nelle democrazie contemporanee, Roma-Bari, Laterza.

Busatto C. (2005), Governança Solidária Local: Desencadeando o processo, Porto Alegre, Prefeitura Municipal de Porto Alegre.

Campos G., Marquetti A. e Pires R. (2008). Democracia Participativa e Redistribuição. Análise de Experiências de Orçamento Participativo, San Paolo, Ed. Xamã.

Caponetto M. (2002), Scenari di progetto identitario. Il caso di Lucca, Firenze, Alinea.

CIDADE (2007), De Olho no Orçamento, Porto Alegre, 11, 21.

D'Albergo E. e Moini, G. (a cura di) (2005), Pratiche partecipative a Roma. Le osservazioni al piano regolatore e il bilancio partecipativo, Roma, Università La Sapienza.

Dagnino E. (2004), "Confluência perversa, deslocamento de sentidos, crise discursiva", In La cultura en la crisis latinoamericana, Buenos Aires, CLACSO.

della Porta D. (2008), "La partecipazione nelle istituzioni: concettualizzare gli esperimenti di democrazia deliberativa e partecipativa", Partecipazione e Conflitto, 0, pp. 15-42.

Diani M. (2008), "Modelli di azione collettiva: quale specificità per i movimenti sociali?", Partecipazione e Conflitto, 0, pp. 43-66.

Douglas M. e Friedmann J. (a cura di) (1997), Cities for citizens: planning the rise of civil society in a global age, Chichester, John Wiley \& Sons.

Dufour D-R. (2005), A arte de reduzir cabeças. Sobre a nova servidão na sociedade ultraliberal, Rio de Janeiro, Companhia de Freud.

Fedozzi L. (2000), O Poder da Aldeia. Gênese e História do Orçamento Participativo de Porto Alegre, Porto Alegre, Tomo Editorial.

Fedozzi L. (2001), Orçamento Participativo: reflexões sobre a experiência de Porto Alegre, Porto Alegre, Tomo Editorial Ltda/FASE.

Fedozzi L. (2007), Observando o Orçamento Participativo de Porto Alegre: análise histórica de dados, perfil social e associativo, avaliação e expectativas, Porto Alegre, Tomo Editorial.

Fedozzi L. e OBSERVAPOA (2009), Observando o Orçamento Participativo de Porto Alegre, perfil social e associativo, avaliação, formação de uma cultura política democrática OBSERVAPOA.

e possíveis inovações (primeiros resultados), Porto Alegre,

Fung A.E e Wright E.O. (a cura di) (2003), Deepening Democracy. Institutional innovations 
in Empowered Participatory Governance, London-New York, Verso.

Ganuza E. (2008), Control político y participación en democracia: los presupuestos participativos, Madrid, Ed. Fundación Alternativas.

Giusti M. (1995), Urbanistica e terzo attore. Ruolo del pianificatore nelle iniziative di autopromozione territoriale degli abitanti, Torino, Harmattan Italia.

Habermas J. (1984), Teoria de la acción comunicativa, Barcelona, Península.

Hettne B. (1996), Le teorie dello sviluppo, Torino, Harmattan Italia.

Hwang H. S. (2008), "Citizen Involvement in Budgeting: The Citizen Participatory Budgeting $(C P B)$. Experience in Korea" consultabile all'indirizzo http://www.opdc.go.th (ultimo accesso effettuato il 3/06/2010).

Jorge F. (2007), Como hacer Presupuesto Participativo. Guía y Herramientas, Santo Domingo, Búho.

King C. S., Feltey KI. e O’Neil Susel B. (1998), “The question of participation: toward authentic public participation in public administration”, Public Administration Review, 58, 4, pp. 317-26.

Langelier S. (2011), Participatory Budgeting in Porto Alegre after the political change, Tesi di Dottorato, Montreal, UQAM.

Menegat E. (1998), "Movimentos sociais e inovações na democratização da gestão urbana em Porto Alegre", Proposta, 78, pp. 48-54.

Muzell P. (2010), Fogaça e o desmonte do Orçamento Participativo, testo disponibile al sito http://rsurgente.opsblog.org/2010/03/10 (ultimo accesso 24/07/2010).

Pellizzoni L. (2008), "Politiche pubbliche e nuove forme di partecipazione". Partecipazione e Conflitto, 0, pp. 93-116.

Pizzorno A. (2008), "I sentieri della partecipazione. Colloquio con Alessandro Pizzorno", a cura di M. Andretta e L. Mosca, Partecipazione e Conflitto, 0, pp. 175-188.

Polletta F. (2006), It was like a fever, Chicago, The University of Chicago Press

Sampaio R. (2010), Participação e Deliberação na Internet: um estudo de caso do Orçamento Participativo Digital de Belo Horizonte, tesi di Master in Comunicazione Sociale, Bolo Horizonte, UFMG.

Sanchez F. (2004), "O Orçamento Participativo de São Paulo (2001-2004): uma inovação democrática", in Avritzer L. (a cura di), A participação em São Paulo, San Paolo, Edunesp, pp. 409-470.

Santos De Sousa B. (2004, a cura di), Democratizzare la democrazia, Troina, Città Aperta.

Schneider A. e Baquero M. (2009), "Instituições governamentais e participação cidadã: finanças públicas inclusivas em Porto Alegre", Revista debates, 3, 2, pp. 183-212.

Sintomer Y. e Allegretti G. (2009), I bilanci partecipativi in Europa. Nuove sperimentazioni democratiche nel vecchio continente, Roma, Ediesse.

Siqueira F. e Campos G. (2009), Notas sobre os deslocamentos participativos em São Paulo.

Talpin J (2007), Schools of Democracy: How Ordinary Citizens Become Competent in Participatory Budgeting Institutions, Tesi di Dottorato, Firenze, EUI.

Villaman M. (2010), "De la Democracia Representativa a la Democracia Participativa".

In Primer seminario presencial Escuela de Politicas de participación Social, Santo Domingo, 15-19/07, Parlocal/Diputación de Malaga.

Wampler B. (2007), Participatory Budgeting in Brazil. Contestation, Cooperation, and Accountability, Penn State University Press. 